\title{
1 Estimating individual level plant traits at scale
}

2 Sergio Marconi $^{1 *}$, Sarah J. Graves ${ }^{2,3}$, Ben. G. Weinstein ${ }^{4}$, Stephanie Bohlman ${ }^{2}$, Ethan P. White ${ }^{4}$

$4{ }^{1}$ School of Natural Resources and Environment, University of Florida, Gainesville, FL, 32611

5 USA

$6{ }^{2}$ School of Forest Resources and Conservation, University of Florida, Gainesville, FL 32603, USA

7 3University of Wisconsin-Madison, Nelson Institute for Environmental Studies, Madison, WI, United

8 States, 53706

9 'Department of Wildlife Ecology and Conservation, University of Florida, Gainesville, FL 32603, USA

10

11

12 Corresponding author: Sergio Marconi, sergio.marconi@weecology.org 


\section{Abstract}

16 Functional ecology has increasingly focused on describing ecological communities based on

17 their traits (measurable features affecting individuals fitness and performance). Analyzing trait

18 distributions within and among forests could significantly improve understanding of community

19 composition and ecosystem function. Historically, data on trait distributions are generated by (1)

20 collecting a small number of leaves from a small number of trees, which suffers from limited

21 sampling but produces information at the fundamental ecological unit (the individual); or (2)

22 using remote sensing images to infer traits, producing information continuously across large

23 regions, but as plots (containing multiple trees of different species) or pixels, not individuals.

24 Remote sensing methods that identify individual trees and estimate their traits would provide the

25 benefits of both approaches, producing continuous large-scale data linked to biological

26 individuals. We used data from the National Ecological Observatory Network (NEON) to

27 develop a method to scale up functional traits from 160 trees to the millions of trees within the

28 spatial extent of two NEON sites. The pipeline consists of three stages: 1) image segmentation,

29 to identify individual trees and estimate structural traits; 2) ensemble of models to infer leaf mass

30 area (LMA), nitrogen, carbon, and phosphorus content using hyperspectral signatures, and DBH

31 from allometry; and 3) predictions for segmented crowns for the full remote sensing footprint at

32 the NEON sites.

33 The $\mathrm{R}^{2}$ values on held out test data ranged from 0.41 to 0.75 on held out test data. The ensemble

34 approach performed better than single partial least squares models. Carbon performed poorly

35 compared to other traits $\left(\mathrm{R}^{2}\right.$ of 0.41$)$. The crown segmentation step contributed the most

36 uncertainty in the pipeline, due to over-segmentation. The pipeline produced good estimates of

$37 \mathrm{DBH}\left(\mathrm{R}^{2}\right.$ of 0.62 on held out data). Trait predictions for crowns performed significantly better 
38 than comparable predictions on pixels, resulting in improvement of $\mathrm{R}^{2}$ on test data of between

390.07 to 0.26 . We used the pipeline to produce individual level trait data for $\sim 5$ million individual

40 crowns, covering a total extent of $\sim 360 \mathrm{~km}^{2}$. This large dataset allows testing ecological

41 questions on landscape scales, revealing that foliar traits are correlated with structural traits and

42 environmental conditions.

44 Keywords: Plant traits, Foliar, Structural, NEON, Hyperspectral, LiDAR, Individual Tree Crown

\section{Introduction}

47 Functional traits are biochemical, physiological and structural characters that influence organism

48 performance or fitness (Nock et al., 2016). They are central to how organisms perform under

49 different environmental conditions, interact with other species, and influence the ecosystems to

50 which they belong (McGill 2006, Dwyer et al., 2017, Collalti et al., 2019). For individual

51 organisms, traits influence core demographic parameters including survival and reproduction. At

52 the species level, traits influence species distributions and how species respond to changes in

53 land use and climate (Pollock et al., 2012). At the ecosystem level, organismal traits influence

54 biogeochemical cycles and habitat availability for other species (e.g., Fisichelli et al., 2015).

55 Given their central importance across multiple levels of organization, understanding how traits

56 vary within and among species, across environmental gradients, and through time is essential to

57 understanding many areas of ecology and predicting how ecological systems will change in the

58 future (McGill 2006, Lawler et al. 2010, Valladares et al., 2014, Diaz et al., 2016). 
59 In trees, two commonly studied groups of traits are specific to (1) properties of leaves (e.g., leaf

60 mass per area, nitrogen and phosphorus concentration) and (2) the size structure of the full tree

61 (e.g. height, dbh, canopy size). These characters hold different information about tree properties

62 and how they link to forest functions. Nitrogen and phosphorus, for example, are fundamental

63 proxies of leaf productivity because of their fundamental role in photosynthesis (Tang et al.,

64 2018); LMA is a widely used indicator of different leaf anatomy and foliar structure strategies

65 (Poorter et al., 2008); and tree height and dbh are indicators of tree structure and growth. Having

66 access to measures of both leaf and structural (or physiognomic) traits for individual trees across

67 the landscape potentially unlocks the ability to explore different dimensions of biodiversity

68 together, investigate how these properties influence each other and affect competition among

69 neighboring trees, and link to tree functions like growth and carbon exchange. However,

70 exploring the links between leaf and structural traits across landscape is challenging, in part

71 because of the differences in the design of their sampling approach.

72 Historically, structural traits are collected for thousands of trees in targeted areas via programs

73 such as the US Forest Inventory and Analysis, whereas studies of leaf chemical traits have relied

74 on collecting samples of a few leaves from a small number of individuals. These values are used

75 to estimate the average trait values for each species and to explore how ecosystem level leaf

76 traits vary biogeographically or through time by assuming that all individuals of a species in a

77 region share the same trait value (Swenson et al., 2010, Clark et al., 2016). This approach is

78 necessary because it is expensive and time consuming to collect individual level leaf trait data,

79 but it fails to describe trait variation within species driven by evolution and plastic responses to

80 the conditions an individual or population experiences (Messier et al., 2017, Niinemets et al,

81 2017, Muller et al. 2010, Nicotra et al. 2010, Albert et al. 2010, Callaway et al. 2003). Moreover, 
82 since the number of leaf trait records is often orders of magnitude smaller than tree structural

83 trait records, discrepancy in their sample size may affect the generality of relationships observed

84 between leaf and structural traits at landscape scales. This limitation is magnified when studying

85 changing environments (across space or time) because of bias in where the data for each species

86 is collected. Data are typically collected in small subsets of the full range of conditions that

87 species experience and are often selected in a biased manner that fits the purpose of the original

88 studies (e.g., selecting individuals of a particular health status, size or species). Measuring traits

89 systematically across geographic gradients would address this limitation, but is not feasible with

90 traditional field methods (Anderson-Teixeira 2015).

91 An alternative approach that allows continuous estimation of traits across the landscape is to use

92 remote sensing data (Kerr \& Ostrovsky, 2003, Homolova et al. 2013, Houborg et al., 2015). For

93 example, (a) hyperspectral remote sensing imagery is used to estimate the chemical composition

94 of sunlit leaves by measuring light absorption and reflectance in the visible and near-infrared

95 spectrum (Asner et al., 2017), and (b) light detection and ranging (LiDAR) is used to measure

96 vertical and horizontal vegetation structure (Andersen et al., 2005). Leveraging remote sensing

97 approaches allows for measuring trait information continuously at landscape scales. Together,

98 LiDAR and hyperspectral data can be used to estimate many of the standard leaf and structural

99 tree traits for trees (Serbin et al., 2014, Singh et al., 2015, Asner et al., 2017, Barbosa et al.,

100 2017).

101 Traditionally, remote sensing applications use either the pixel (the smallest resolution component

102 of the image, Audebert et al., 2019) or the plot (a region of space typically containing multiple

103 individuals, Singh et al., 2015, ) as the fundamental unit. This is a natural result of the structure

104 of the remote sensing data combined with the difficulty of linking individual crowns in remote 
105 sensing images to field data, especially for small crowns harder to detect with airborne

106 technology (Jakubowski et al., 2013). However, pixel or plot-based output results in a

107 disconnect between the remote sensing analysis and one of the fundamental biological units: the

108 individual (Liu et al., 2016, De Angelis, 2018, Marconi et al. 2019). Individual plants reproduce,

109 interact with their neighbors, and exhibit plastic responses to environmental conditions at the

110 individual scale. Populations of individuals evolve in response to natural selection. As a result,

111 our understanding of many biological processes is grounded in the individual and many field-

112 based survey methods focus on collecting data with individual trees as the primary unit of

113 measurement. While forest inventories hold information of the individual trees ineach plot

114 (Newnham et al., 2015), plot level estimates from remote sensing typically do not include

115 information about the relative distribution of individuals and their traits within the plot, thus

116 reducing the amount of information about community structure. To fully understand how traits

117 vary across space and time, and are determined by biological processes, it is important to

118 develop approaches linking these fundamental characteristics to individual trees in ways that can

119 be applied at scales of hundreds of $\mathrm{km}^{2}$.

120 Despite its importance for biological research, few studies have attempted to model both leaf and

121 structural traits using remote sensing at the individual level over landscape scales (but see

122 Chadwick \& Asner 2016, Martin et al., 2018). Even when crown level models are developed, the

123 resulting leaf trait predictions are made for pixels, not crowns, due to the challenges in crown

124 segmentation, poor spatial resolution of hyperspectral data, or misalignment between LiDAR and

125 hyperspectral data (Blaschke, 2010). Similarly, there are few studies estimating structural traits

126 (like crown height and area) at crown level, with traditional methods predicting tree height and

127 cover at the plot level (Kaartinen et al., 2012). Perhaps as a result of these differences, structural 
128 and chemical leaf traits are not currently predicted together at large scales. Consequently,

129 ecology lacks the large scale individual level trait estimates that are necessary to fully understand

130 tradeoffs between leaf and tree structural traits, and to explore how trait variability relates to

131 species and the environment. Data from NEON Airborne Observatory Platform NEON (AOP)

132 provide co-registered, georeferenced, and atmospherically corrected high resolution

133 hyperspectral data and LiDAR, whose integration represents a great opportunity to circumvent

134 these challenges.

135 To address this gap, we developed a pipeline for making crown level trait predictions at scales of

$136 \sim 400 \mathrm{~km}^{2}$ with associated uncertainties on both crown segmentation and trait estimation.

137 Building on Chadwick \& Asner (2016) and Martin et al. (2018), we: (1) identify individual

138 crowns in remote sensing imagery that are associated with field-based trait measurements; (2)

139 build models relating the remote sensing data to the field-based trait measurements; and (3)

140 apply those models to estimate trait values and examine patterns of tree structural and chemical

141 traits from individual to landscape scales. We advance the state of the art (Chadwick \& Asner

142 2016, Martin et al. 2018) in this pipeline by using crown-level models and comparing them to

143 pixel- and crown average-level models, directly estimating uncertainty in trait predictions using

144 likelihoods, and predicting traits at the crown-level. Finally, derived data products on the

145 location, size, shape, and leaf traits of millions of individual trees distributed over tens of

146 thousands of hectares.

$147 \quad$ 2. Methods

148 In our pipeline for predicting crown level leaf and structural traits from remote sensing we used:

149 1) field measurements of traits for building and evaluating models; 2) data on the shape and 
150 location of individual tree crowns (ITCs) for building accurate models and assessing uncertainty

151 in crown segmentation algorithms; and 3) high resolution remote sensing LiDAR (for crown

152 segmentation and estimation of structural traits) and hyperspectral data (for estimation of leaf

153 chemical traits). To obtain these components, we combined National Ecological Observatory

154 Network's (NEON) airborne observatory data with field data we collected at NEON sites on leaf

155 traits as well the location and shape of individual tree crowns.

\subsection{Site descriptions}

157 The study was conducted at two core terrestrial NEON sites; Ordway Swisher Biological Station

158 in Florida (OSBS, NEON Domain 03) and the Oakmulgee Management District of Talladega

159 National Forest in Alabama (TALL, NEON Doman 08). The two sites (Appendix S1: Figure S.1)

160 have a mix of deciduous, evergreen, and mixed forest types (Homer et al., 2012). Upland areas at

161 both sites are dominated by fire-tolerant oaks and pine species, primarily longleaf pine (Pinus

162 palustris). The longleaf pine at OSBS forms open stands whereas the longleaf pine canopy at

163 TALL is more closed. Lowland areas near lakes or wetlands (OSBS), and riparian areas (TALL)

164 are dominated by closed canopy hardwood forests (Beckett and Golden 1982, $\underline{\text { Cox and Hart }}$

$165 \underline{2015})$.

167 All aerial remote sensing data products were provided by the NEON Airborne Observation

168 Platform (NEON-AOP, Table 1). We used data from the May 2014 flight for OSBS, and the

169 June 2015 flight for TALL. We used the raw L1 data products: (1) "classified LiDAR point

170 cloud", and (2) "hyperspectral surface reflectance" data, orthorectified and atmospherically 
172 reduce the effects of non-lambertian diffuse scattering, we applied the topographic and

173 bidirectional reflectance distribution function (BRDF) corrections by adapting scripts from the

174 HyTools repository (https://github.com/EnSpec/HyTools-sandbox) to our dataset. The LiDAR

175 data consist of $3 \mathrm{D}$ spatial point coordinates $\left(4-6\right.$ points $\left./ \mathrm{m}^{2}\right)$ which provides high resolution

176 information about crown shape and height. These data are released in $1 \mathrm{~km} \times 1 \mathrm{~km}$ tiles.

177 Hyperspectral reflectance data consist of $1 \mathrm{~m}^{2}$ spatial resolution images with 426 channels (or

178 bands), each one collecting the magnitude of reflectance in $5 \mathrm{~nm}$ wide interval of wavelengths,

179 ranging from visible to near infrared light (from 350 to $2500 \mathrm{~nm}$ ). These images were provided

180 as multiple $\sim 15 \mathrm{~km}$ x $0.8 \mathrm{~km}$ flight lines with a total area of $\sim 215 \mathrm{~km}^{2}$ in OSBS, and $\sim 145 \mathrm{~km}^{2}$ in

181 TALL. The hyperspectral images were provided as "prototype" data, pre-processed differently

182 than post 2017 data, and delivered on hard drives. Prototype airborne data showed misalignments

183 between LiDAR and hyperspectral products, (as well as across hyperspectral flightpaths), on the

184 scale of 1-2 meters (Marconi et al. 2019, Appendix S1: Figure S.6), primarily affecting pixels at

185 crown borders. Despite the prototype data being potentially of lower quality than the newer

186 NEON AOP data, we used it to match the collection dates of the field data. The only difference

187 with current L1 and L3 data is in the nomenclature of the .h5 data structure, making the methods

188 presented here suitable with more recent NEON data.

191 During this project, leaf trait data collected by the NEON Terrestrial Observation System (TOS)

192 were not available. Instead, we used a dataset of leaf samples collected for 157 trees, many of 
193 which were near NEON inventory plots that are randomly located across the study site and

194 stratified by land cover type. The sampled trees were located away from major roads, had crowns

195 visible from airborne aircraft and identifiable in the image, and had sunlit branches that were

196 accessible for leaf collection. Trees were not sampled within NEON plots to avoid disturbing

197 NEON's long-term monitoring efforts. As wide a range of species as possible were collected,

198 including some rarer species that occured far from NEON plots (Appendix S1: Figure S.2).

199 Trait data were collected in the early part of the peak growing season in 2015. Specifically, 81

200 individual trees (of 17 species) were sampled from OSBS in May-June 2015, and 78 individuals

201 (26 species) in July 2015 from TALL. Leaves were sampled from the sunlit portion of the

202 canopy with a shotgun. Immediately after collection, the leaves were placed in a labeled plastic

203 bag and stored in a cooler until they could be processed in the field lab within 2-4 hours of

204 collection. The collected leaves were randomly sampled for further processing in two ways.

205 First, a sample of leaves (at least 20 grams of fresh leaves) was analyzed for nitrogen $(\% \mathrm{~N})$,

206 carbon $(\% \mathrm{C})$, phosphorus $(\% \mathrm{P})$ by weight, according to standard protocols, with the exception

207 that petioles were removed (Murphy and Riley 1962; Cornelissen et al. 2003). Second, a sample

208 was processed for LMA using the Carnegie Institute for Science spectronomics protocol

209 (https://gao.asu.edu/spectranomics, Asner et al., 2011). Whole leaves were weighed then

210 scanned on a flatbed scanner to determine leaf area. The leaves were then dried at $60 \mathrm{C}$ for at

211 least 72 hours and reweighed to get the dry leaf mass. For needle-leaf species, a sample of

212 individual needles (at least 3 fascicles per sample) was scanned and weighed. The needled

213 dimensions of a subset of the samples were also measured with calipers to calculate total surface

214 area. These measurements showed good agreement with the projected surface area from the 
215 scans $\left(\mathrm{R}^{2}>0.75\right)$. This data and complete metadata will be added to TRY database v.6 (Kattge et

216 al., 2020).

217 Individual trees were mapped in the remote sensing images using a field tablet and GIS software.

218 Mapping was done on 2014 imagery for OSBS, and on 2015 imagery for TALL. This process

219 involved mapping individual tree crowns on the hyperspectral image in the field to ensure the

220 sampled trees matched directly with image pixels (Graves et al., 2018). This individual tree

221 crown (ITC) data provides the most accurate link of field measurements with pixels from remote

222 sensing spectral data and was used to quantify uncertainty in crown segmentation algorithms.

224 We developed a modular pipeline based on three steps: (1) build and evaluate crown

225 segmentations from LiDAR data (section 2.6); (2) develop an ensemble of statistical models to

226 infer leaf mass per area (LMA, $\left.\mathrm{g} \mathrm{m}^{2}{ }^{2}\right)$, nitrogen $(\% \mathrm{~N})$, phosphorus $(\% \mathrm{P})$, and carbon $(\% \mathrm{C})$ per

227 tree from hyperspectral data (section 2.5), and models to estimate structural traits [diameter at

228 breast height, DBH (cm), crown area, CA $\left(\mathrm{m}^{2}\right)$ and stem height, $\left.\mathrm{H}(\mathrm{m})\right]$ from LiDAR data; (3)

229 make predictions for every individual tree crown in both NEON sites. For each crown, we also

230 extracted values of elevation, slope and aspect provided as NEON AOP data products (Table 1),

231 aiming to build a comprehensive dataset including topographic, leaf chemical and tree structural

232 traits for any tree detected within the AOP footprint. We limited our analysis to individual tree

233 crowns taller than 2 meters and wider than $1 \mathrm{~m}^{2}$. Since the field traits dataset was for sunlit

234 foliage, we predicted traits only from the upper portion of the canopy. The structure of the

235 pipeline presented in this paper is summarized in Figure 1. 


\subsection{Leaf chemistry model}

237 After correcting the hyperspectral data with the bidirectional reflectance distribution function

238 and topographic correction, we extracted all pixels within the boundaries of the field-delineated

239 ITCs. Shadowed or low vegetation pixels within the ITCs were removed using thresholds for

240 both a near infrared band (reflectance in $860 \mathrm{~nm}<0.3$ ) and the Normalized Difference

241 Vegetation Index (NDVI < 0.7) (Appendix S1: Figure S.3) Colgan et al., 2012, Graves et al.,

242 2016). We normalized the spectral values for each pixel by dividing each spectral vector by its

243 root sum of squares. We used this method to further reduce the effect of peripheral light and

244 shadows within each crown (Singh et al., 2015, Feilhauer et al., 2010).

245 Field data were split at the tree level, and stratified by species, into training $(n=115)$, validation

$246(\mathrm{n}=18)$, and test sets $(\mathrm{n}=24)$. Since the two sites have similar species composition, we

247 aggregated the two datasets to build a joint model. As is common for trait studies, our field data

248 on foliar traits was averaged to a single value for each individual tree. Most algorithms require

249 associating a single vector of predictor variables (i.e. the spectra) to a single response value (e.g.

250 tree crown or plot). However, individual crowns contain multiple pixels, and crowns vary in the

251 number and quality of these pixels. In each crown, some pixels will be better for linking traits to

252 hyperspectral signatures because they reflect light primarily from leaves, whereas other pixels

253 include reflectance from branches, understory, or ground. To address this, we used a bagging

254 approach (Song et al., 2013) that takes advantage of different pixel characteristics by training,

255 weighting, and ensembling models fit to different subsets of pixels. This approach weights the

256 predictions from models fit to different pixels to produce a more generalizable and accurate

257 representation of the relationship between foliar traits and their spectral signatures. To capture

258 the range of possible models from different subsets of pixels in each crown, we randomly 
sampled one pixel from each training crown 1000 times, and used the resulting 1000 vectors of

260 pixels (one pixel for each crown in the dataset) to build 1000 independent partial least squares

261 generalized linear regression (PLS-GLR) models (Bastien et al., 2005, Bertrand et al, 2014).

262 Instead of the regular PLS regressions used in most trait modeling (e.g., Singh et al., 2015, Wang

263 et al., 2020, Chadwick et al., 2018) we used PLS-GLR because it uses maximum likelihood

264 estimation to calculate the regression parameters. This is an improvement over current

265 approaches because it: (1) allows the calculation of AIC for model averaging (Burnham \&

266 Anderson 2002); (2) provides a robust measure of uncertainty in the form of a prediction interval

267 (Christoffersen, 1998), which allows estimating the range of out of sample predictions rather

268 than the range of mean response; and (3) does not require bootstrapping, making the method

269 more scalable. We used a log-normal link function for all models to reflect the fact that all traits

270 are positive numbers. We treated site (OSBS versus TALL) as a one hot encoder fixed effect

271 (Harris \& Harris, 2010) to account for site specific ancillary conditions. The number of

272 components included in each model were determined using 5-fold cross-validation (CV) using

273 the PRESS statistic (Tapley, 2000) on the training set. Models for each leaf trait were trained

274 independently.

275 We compared four modeling strategies that varied in how the models were developed and how

276 the models were applied for testing (Appendix S1: Table S.1). The models are labelled based on

277 how the model was applied as "pixel based" (applied to individual pixels) or "crown based"

278 (applied to segmented crowns). The models were built as follows: 1) a pixel-based approach

279 with the spectra of a single pixel randomly extracted from each crown (SPM). This approach

280 represents the case in which only the coordinates of the leaf sample are available, and spectral

281 information can be extracted by sampling from a pixel corresponding to the stem or leaf location; 
2) A pixel based approach (hereafter referred as the "ensemble pixel based model" (or EPBM) that included information on crown identity by labelling each pixel with an individual crown identifier and using it to ensemble a selection of 100 SPM models using multi-model averaging based on delta AIC (Burnham \& Anderson 2002). In this step, we selected the 100 models for each trait that best performed on the independent validation dataset $(n=15)$. This step was

287 fundamental to: (a) drop models that performed worse than chance $\left(\mathrm{R}^{2}<0\right)$ and therefore held 288 not meaningful relationships; (b) massively reduce the computational resources required to scale 289 predictions to hundreds of $\mathrm{km}^{2}$.This approach requires crown boundaries information for training 290 but not for application, since it applies to individual pixels; 3) a simple crown average approach, 291 hereafter "Crown Average Spectra" (CAS), where each individual tree was represented by the 292 average of the spectra across all green pixels (i.e. pixels with NDVI $>0.7$ and NIR $>0.3$ ) within 293 the crown polygon; 4) A crown average approach, that we refer to as the "Crown Ensemble 294 Aggregation Model" (CEAM), consisting on averaging predictions from the EPBM for all sunlit 295 pixels belonging to individual tree crown polygons. We tested the performance of each approach 296 in two ways, on pixels extracted from (1) ground delineated crowns (Graves et al., 2018), and (2) 297 algorithmically delineated crowns (Silva et al., 2016). This step was fundamental to quantify the 298 effect of uncertainty in crown detection and segmentation on predicting leaf traits at crown level 299 across the landscape where no field delineated crowns are available.

300 All models were tested on the 24 crowns withheld in the test dataset. The test data were not used 301 at any phase of the fitting or the ensemble process. Accuracy was evaluated using the predictive 302 coefficient of determination $\left(\mathrm{R}^{2}\right)$ and the root-mean-square error (RMSE). The coefficient of 303 determination produces values between 1 and negative infinity, where negative values indicate

304 that the model predictability is lower than the sample average. As such, negative $\mathrm{R}^{2}$ values 
305 indicate that the statistical model did not learn any meaningful information from the data. A

306 value of 1 indicates that predicted values perfectly match observations. We evaluated the

307 uncertainty of predictions for each model using the coverage of the $95 \%$ prediction interval

308 (95PI). The prediction interval is the range of values that is expected to contain $95 \%$ of the

309 observed data points, and therefore a model with good estimates of uncertainty should have

310 approximately $95 \%$ of the test data falling within this range. Since the CEAM was generated by

311 the ensemble of the 100 best SPMs, we estimated the 95PI for CEAM predictions by averaging

312 the error functions for the same 100 SPMs. We used the same data split, data transformation and

313 PLS-GLR parameterization for all models. For pixel-based estimations (SPM and EPBM), we

314 compared ground measures of LMA, N, C, and P with predictions from each pixel in the test

315 dataset. For crown-based estimations (CAS and CEAM), we averaged pixel-based predictions

316 belonging to all of the pixels in the crown. The same rationale was used for comparing pixel and

317 crown-based uncertainty.

319 We used the lidR R package (Roussel \& Auty, 2017) to process point cloud LiDAR data to

320 create a $0.5 \mathrm{~m}^{2}$ resolution canopy height model (CHM) and produce algorithmically delineated

321 crowns. Despite there was little difference to the $1 \mathrm{~m}^{2}$ resolution of NEON CHM, we chose an

322 higher resolution CHM to produce smoother polygons and leverage the information in regions

323 where the point cloud was more dense. We used the CHM to determine the number of trees in

324 the scene (i.e. tree detection) using local maxima filtering (Popesco et al., 2004). We tested three

325 alternative methods for crown segmentation (Dalponte \& Coomes 2016, Silva et al., 2016, and a

326 watershed algorithm as in Barnes et al., 2014) and chose the best performing one to generate 
crown boundaries (Appendix S1: Table S.2, Appendix S1: Section 1). To evaluate accuracy of

328 crown detection and segmentation on the targeted landscapes, we calculated: (1) an estimate of

329 precision from all predicted crowns whose boundaries overlapped with the field ITCs; (2)

330 pairwise Jaccard index coefficient (Real \& Vargas, 1996), which represents the intersection over

331 union between the areas of two polygons, and is the standard benchmarking metric for image

332 analysis (Rezatofighi et al., The Jaccard index was calculated by comparing ITCs collected in

333 the field with the single most overlapping predicted crown (Marconi et al., Field delineated

334 crowns that do not overlap with any crown segmented by the algorithm were labelled as

335 undetected. 2019). We estimated tree structural traits from the derived polygons and the CHM.

336 Crown area (CA) was calculated from the polygon geometry using the geoPandas python

337 package (https://geopandas.readthedocs.io/). Tree height (H) was extracted from the CHM as the

338 maximum height within each ITC. Diameter at breast height (DBH) was calculated using an

339 allometric regression model relating the log-transformed DBH taken from the NEON woody

340 plant vegetation structure data to the log-transformed height and canopy area of the matching

341 algorithmically delineated crowns for 566 individual stems. Delineated crowns were matched to

342 field-mapped stems in the NEON dataset visually (Appendix S1: Table S.3).

\subsection{Building individual-level derived data for full flight paths}

344 Each remote sensing image was split into $1 \mathrm{~km}^{2}$ tiles to optimize computational resources and

345 allow parallelization on hundreds of cores. We pre-processed each tile using the same filters used

346 for developing the models. To make predictions we used the EPBMs ensemble models to

347 produce rasters of LMA, \%C, \% $\mathrm{P}$ and $\% \mathrm{~N}$ predictions and the 95PI for each suitable pixel and

348 averaged them to crown level by using algorithmically delineated crowns. Crown-based

349 predictions were achieved by averaging the values of all suitable pixels within the corresponding 
350 predicted ITC boundaries. For those areas where the ITC overlapped with more than one flight

351 path (flight paths overlap by $\sim 30 \%$ ), we averaged the crown-based predictions from both flight

352 paths.

353 We stored flight-paths level maps of traits into raster data-products. The crown level dataset was

354 then compiled as a comma delimited file containing all the geometry information to rebuild

355 polygon shapes and locations. The data is distributed in a Zenodo archive

356 (http://doi.org/10.5281/zenodo.3232978).

3. Results

359 We chose the crown segmentation algorithm described in Silva et al. (2016) (Appendix S1:

360 Table S.2) to produce algorithmically delineated crowns. The approach detected $\sim 88 \%$ of the

361 field crowns (Appendix S1: Table S.5), but showed lower accuracy in estimating the shape and

362 size of the canopies for individual trees, with Jaccard Index ranging between 0 (for undetected

363 trees) and 0.81 , with an average of 0.35 . Crowns identified by the algorithm were generally

364 larger than those delineated in the field, resulting in overestimated crown areas (especially for

365 smaller trees) and weak correlations between field data and algorithmic crown areas (Figure 2).

366 Low goodness of fit in predicting Crown Area (CA) was exacerbated by uncertainty in alignment

367 with field and remote sensing data. For example, field crowns were delineated on the

368 hyperspectral images to incorporate only the pure pixels of the crown (Graves et al., 2018,

369 Appendix S1: Figure S.4, Appendix S1: Figure S.5) leading to potentially underestimating the

370 full extent of tree crown size. Moreover, visual assessment of paired field and algorithmically

371 delineated crowns shows shifts by 1-2 meters that likely result from imperfect alignment

372 between LiDAR and hyperspectral data (Appendix S1: Figure S.6; Marconi et al. 2019), further 
373 affecting uncertainty in field to algorithmically estimated crowns. Estimates of other structural

374 traits from the algorithmic tree crowns were better than crown area estimates. Height showed the

375 highest correspondence between field and remotely sensed measures $\left(R^{2}=0.90\right.$ for trees higher

376 than $3 \mathrm{~m})$. Despite low accuracy in its predictions, CA showed a significant effect in estimating

377 DBH from LiDAR (Appendix S1: Table S.3). However, tree height was the most important

378 variable in predicting DBH, which therefore resulted in good estimates for both sites $\left(0.62 \mathrm{R}^{2}\right)$,

379 in the range of other recent applications (0.59 in Dalla Corte et al., 2020, 0.62 to 0.83 from Yao

380 et al., 2012).

381 We compared the four methods - two applied to pixels (the single pixel methods, SPM, and

382 ensemble pixel methods, EPBM) and two applied to crowns (the crown average, CAS and the

383 crown based ensemble methods, CEAM) - using RMSE. When tested on pixels extracted from

384 field delineated crowns, CEAM performed the best for \% $\mathrm{N}$ and $\% \mathrm{P}$ (RMSE of 0.20 and 0.026 ),

385 and CEAM and EPBM performed equivalently for LMA (RMSE of 40.3 and 40.8 respectively)

386 (Figure 3A, Appendix S1: Figure S.7). CEAM explained 75\% of the variance in LMA, 66\% of

387 the variance in $\% \mathrm{~N}, 46 \%$ of the variance in $\% \mathrm{P}$, and $41 \%$ of the variance in $\% \mathrm{C}$ (Appendix S1:

388 Table S.4, Appendix S1: Figure S.8). These results were comparable to those obtained by Martin

389 et al. (2018) for trees in Borneo $(71 \%, 46 \%, 44 \%, 48 \%$ respectively for LMA, \% N, \%P, \%C).

390 Despite "site" ancillary information was an important feature for all models, its influence on

391 traits predictions was always relatively low compared to reflectance (as shown by the models'

392 parameters, Appendix S1: Figure S.9). The root mean squared error (normalized by traits

393 observations range, NRMSE) was always between 8 and 16\% of the range of the field

394 observations, meeting the quality threshold recommended by Singh et al. (2015). 
395 The ensemble approaches (CEAM and EPBM) performed better than methods based on pixels as

396 the fundamental unit (SPM) or simple averaging of all green pixels in a crown (CAS) when the

397 assessment was on field delineated crowns (Appendix S1: Table S.4; Figure 3A). Few individual

398 SPMs performed better than the EPBM ensembles (Appendix S1: Figure S.10) and when

399 individual pixel models did outperform the ensemble, they usually were not among the best SPM

400 models (i.e., the models with the lowest delta AIC in the validation). This suggests that EPBM

401 provides the best method for making out-of-sample predictions at the pixel level. CEAM

402 generally produced the best estimates of uncertainty. CEAM 95PI showed an average coverage

403 of $95 \%$ of held out observations, CAS 94\%, EPSM 91\%, Plot and SBM 90\%, with the ideal

404 value being 95\% (Figure 3D).

405 The CEAM approach performed best when making predictions using pixels extracted from

406 algorithmically delineated crowns (Figure 3). Compared to when crown boundaries are collected

407 from the field, the accuracy of predictions using algorithmically delineated crowns was reduced

408 due to the uncertainty associated with crown segmentation. However, CEAM showed the lowest

409 reduction in accuracy compared to the other approaches ( $\triangle \mathrm{NRMSE} \sim 2 \%$, Figure $3 \mathrm{C})$, resulting

410 in the lowest NRMSE for all traits (Figure 3B).

411 Scaling algorithmic crown segmentation and trait estimation to the full extent of the NEON

412 remote sensing data yielded trait predictions for approximately 5 million canopy trees for the two

413 sites combined (Figure 4, Appendix S1: Figure S.11, S.12, S.13). Landscape patterns in traits are

414 evident, including east-west gradients in LMA, \%N, and \%P at OSBS (Figure 4). At TALL,

415 lower LMA and higher $\% \mathrm{~N}$ and $\% \mathrm{P}$ are found in a dendritic pattern associated with the stream

416 network (Appendix S1: Figure S.12). Some traits show a bimodal distribution at each site, which

417 is probably related to differences in needleleaf versus broadleaf species but would need to be 
418 further tested with trait estimation coupled with species predictions. On average, OSBS showed

419 higher $\% \mathrm{~N}$ and \%P compared to TALL (Figure 5). Distributions of LMA, \%N, and \%P in OSBS

420 shifted to higher values than TALL, following patterns observed from the field data (Figure 5,

421 Appendix S1: Figure S.15). Assessing correlations between estimated structural traits, leaf traits,

422 and abiotic environmental conditions showed strong correlations between LMA, \% N, and \%P,

423 consistent with the leaf economic spectrum (Wright et al., 2004) (Appendix S1: Figure S.14). Of

424 the environmental variables, elevation had the strongest relationship with leaf traits with leaf $\mathrm{N}$

425 and P decreasing and LMA increasing with elevation (Figures S.15, S.16). Leaf traits and tree

426 structure were correlated at OSBS (e.g. LMA with tree H, figure 5B) but not TALL.

\section{Discussion}

428 The individual organism is one of the fundamental units of biology. As a result, studying the

429 distribution of individuals and their traits across space and through time is central to many

430 aspects of ecology. However, collecting individual level data at the large scales required for

431 many ecological questions is challenging. To address this limitation we develop a fully

432 automated modular pipeline to link remote sensing products from the National Ecological

433 Observatory Network (NEON) to data collected in the field, convert the remote sensing data into

434 estimates of the leaf and structural traits for all canopy trees detected at landscape scales, and

435 estimate the traits for millions of individual trees in an open and accessible format (Cassey et al.,

436 2006, Hampton et al., 2016) for use by the broader scientific community.

437 We found that modeling and predicting leaf traits at the individual crown level resulted in

438 improved accuracy and uncertainty in the predictions compared to pixel based approaches

439 (Figure 3). Linking pixels to crowns allows the ensembling of models built from the different 
440 pixels making up the crowns. Different pixels contain different combinations of leaves, branches,

441 understory and ground, which affects the underlying chemometric relationship between foliar

442 traits and their spectral signature. Weighted ensembling provides one way to address this, by

443 allowing the models to identify the best combinations of pixels for relating traits and

444 hyperspectral signatures. Aggregating pixel predictions to the crown level may also reduce the

445 influence of outlier pixels. Our method produced models with predictive power comparable to

446 two other crown-based estimation methods (Barbosa and Asner, 2017; Martin et al., 2018),

447 suggesting that the performance of these approaches may generalize beyond the current study. In

448 addition to providing robust leaf trait estimates, crown level methods allow the simultaneous

449 estimation of structural traits, allowing these two sets of traits to be analyzed together at large

450 scales.

451 2019). This is likely because height is directly measured by LIDAR and height was the most

452 important factor in the allometric models used to predict DBH. 2018, Jucker et al. 2016). While

453 our crown-based methods were effective for estimating a number of leaf and structural traits,

454 there is substantial uncertainty even for the best performing traits. Quantifying this uncertainty

455 provides information on the range of likely trait values for each individual and allows this

456 uncertainty to be propagated when using these derived data values to test scientific hypotheses

457 (Miller-Gulland and Shea, 2017). We used methods for leaf trait estimation that allowed us to

458 estimate uncertainty (pls-GLR) and the crown-based method (CEAM) provided the best

459 uncertainty estimates (Figure 3, Appendix S1: Table S.4). Current methods for delineating

460 crowns do not include explicit measures of uncertainty (Dalponte \& Coomes 2016, Silva et al.,

461 2016). It is important for future methods to address this limitation because comparisons to field

462 data suggested high uncertainty in segmentation. 
463 One of the challenges for crown level approaches is that they rely on crown segmentation

464 algorithms to identify the location and size of individual trees. While we used the best

465 performing crown segmentation the algorithm from a recent methods competition (Marconi et al.

466 2019) and had reasonable correspondence between the presence of an algorithmic crown and

467 each field crown, the algorithmic crowns averaged only 35\% overlap in area with the most

468 similar field delineated crown. Heterogeneity in point cloud density and misalignment between

469 lidar and hyperspectral sensors could contribute to misalignment between field and

470 algorithmically delineated crowns (Marconi et al, 2019, Kamoske et al., 2019, Appendix S1:

471 Section 1). Despite this uncertainty, estimates of DBH and height were well correlated with field

472 values. Therefore data derived from our pipeline should be useful for assessing estimates of

473 individual tree biomass by applying allometric functions linking biomass to DBH and height

474 (Graves et al. Crown area was more susceptible to the segmentation uncertainty, but is also more

475 sensitive to small errors in segmentation and CHM resolution (Appendix S1: Section 1).

476 Uncertainty in crown segmentation can also have cascading impacts on the estimation of leaf

477 traits, which was tested by comparing results from field versus algorithmically delineated tree

478 crowns. Prediction accuracy is generally lower when using algorithmically delineated crowns

479 because the pixels used for making predictions both include pixels that are not in the true crown

480 and exclude pixels that are in the true crown. However, these decreases in accuracy were

481 generally quite small, with decreases in NRMSE of $<0.05$ across all methods. The crown-based

482 ensemble method (CEAM) was particularly robust to this uncertainty, with the smallest increases

483 in NRMSE and all traits maintaining NRMSE below Singh et al. (2015)'s threshold (Figure 3C,

484 Appendix S1: Table S.4). This robustness may result because the weighted ensembles in CEAM 
485 provide the ability to weight pixels algorithmically, allowing it to ignore pixels from outside of

486 the true crown.

487 Generating derived individual level data on leaf and structural traits at the landscape scale allows

488 trait patterns at these scales to be effectively assessed. While a complete analysis of the spatial

489 distribution of tree traits is beyond the scope of this paper, our results showed some general

490 patterns worth future investigations. LMA, \%N, and \%C showed bimodal distributions at both

491 sites (with \% N peaks particularly close in OSBS), likely because pines and oaks, the most

492 common needleaf and broadleaf genera respectively at these two sites, occupy distinct regions of

493 the worldwide Leaf Economic Spectrum (LES)(Wright et al., 2004). Correlation patterns

494 between LMA and \%N, \% N and \%P, and LMA and \%P match the global scale patterns observed

495 globally in the LES. Despite the limited number of species and geographical extent, both sites

496 showed rangeand spread of LMA, \% N and \%P overlapping with most of the global range of the

497 worldwide LES tradeoffs (Appendix S1: Figure S.14). This suggests that variation in the local

498 environment could be driving large intra-species variability of leaf traits, while conserving the

499 general trade-offs observed across species (Asner et al., 2016). Among the environmental

500 variables we tested, elevation showed the strongest correlation with leaf traits (Appendix S1:

501 Figure S.16, Appendix S1: Figure S. 17), possibly because elevation represents a proxy of

502 different soil conditions in these sites, which can affect both species distributions and leaf traits

503 (Walter \& Gerlach, 2013). For example, small differences in elevation at OSBS often means

504 transitioning from drained sandhill (that favor pines) to marshy and richer soils that favor the

505 establishment of large-crowned broadleaf species, rich in foliar \% $\mathrm{N}$ and \% $\mathrm{P}$ (Bodker et al.,

506 2015). 
507 Producing derived data at the individual level also facilitates landscape scale assessments of

508 relationships between leaf and structural traits at the level of individual organisms. For example,

509 although the two sites have similar species composition, our results showed different

510 correlational patterns between structural (height and $\mathrm{DBH})$ and chemical traits (LMA, \% N, \%P,

$511 \%$ ) (Figure 5, Appendix S1: Figure S.17), especially the relationship between height and LMA

$512(\mathrm{r}=0.24$ in OSBS, $\mathrm{r}=-0.08$ in TALL). Possible explanations for these relationships may be

513 related to differences in management histories across patches of the landscape that can influence

514 species assembly, successional stages, which are important determinants of tree size and leaf

515 traits (Sameulson and Stokes 2012; Ishida et al. 2005). Our pipeline, integrated with further

516 remote sensing derived information (e.g. species identities) and local history (e.g. management

517 and fire history) could be used to address how these drivers affect local distribution of plant

518 traits, their trade-offs, and their effects on the ecosystems across a multitude of landscapes.

519 Our crown-based approach to modeling and predicting tree traits produces individual data similar

520 to that collected in the field. This approach has a number of benefits. First, it will make data

521 integration with field-based forest and trait surveys easier because both derived remote sensing

522 data and field surveys will be composed of the same fundamental unit (individual trees). Second,

523 crown-based approaches are likely better for aligning trait data across years. The same pixel in

524 two consecutive years could vary significantly in a trait because of small errors in spatial

525 alignment of pixels through time, whereas large crown-level regions will be more robust to small

526 errors at the edges of the crown. Moreover, multi-temporal and multi-sensor images can be

527 potentially leveraged to align and improve segmentation for crown objects captured in the same

528 scene (Bovolo \& Buzzone, 2017, Sumbul et al., 2020). Finally, this approach allows a more

529 compact representation of derived trait data in tabular formas spatial polygons instead of rasters. 
530 While this will not be the best representation for all analyses, for individual level analyses it

531 results in vastly reduced storage computational requirements compared to raster data.

532 Thanks to the modular nature of our approach, crown segmentation can easily be substituted with

533 methods based on RGB (Weinstein et al., 2020) or hyperspectral imaging (Dalponte et al., 2016)

534 when LiDAR data is poor or not available. Yet, individual level approaches are limited by data

535 availability and are not suitable for addressing ecological questions at continental to global scale.

536 High resolution airborne remote sensing is still limited to relatively few sites, while the

537 resolution of AVIRIS or satellite data is more appropriate for plot level analyses (e.g., Singh et

538 al. 2015, Martin et al. 2018, Ma et al. 2019). However, these two approaches can be potentially

539 integrated to scale sub-pixel properties from satellite data, and merge the gap between local,

540 regional and global scale ecological information, and better address emergent cross-scale

541 ecological questions related to variation of leaf traits, diversity and functions (Carmona et al.,

542 2016).

543 The data produced by our individual level pipeline could be extended by including predictions

544 for species identity, other leaf and structural traits, environmental variables, management, or

545 disturbance. Moreover, our pipeline can potentially be used to extract ecological information for

546 every tree that can be detected across all NEON AOP sites for the full life of the observatory.

547 This will produce a publicly available, spatially explicit database of detailed ecological

548 information for hundreds of millions of trees across the US that could be fused with other

549 continental data (e.g. Forest Inventory and Analysis) and integrated to area based analyses from

550 satellite data, to address cross scale functional ecological questions

551 (http://doi.org/10.5281/zenodo.3232978). Such data could be used to further understand the 
552 biology behind trait tradeoffs and investigate cross scale ecological processes and patterns from

553 individual to landscape to continental scale.

555 This work was supported by the Gordon and Betty Moore Foundation's Data-Driven Discovery

556 Initiative through grant GBMF4563 to E.P. White and by the National Science Foundation

557 through grant 1926542 to E.P. White, S.A. Bohlman, A. Zare, D.Z. Wang, and A. Singh; by the

558 NSF Dimension of Biodiversity program grant (DEB-1442280) and USDA/NIFA McIntire-

559 Stennis program (FLA-FOR-005470) to S. A. Bohlman; by the University of Florida

560 Biodiversity Institute (UFBI) and Informatics Institute (UFII) Graduate Fellowship to Sergio

561 Marconi. There was no additional external funding received for this study.

\section{Description of author's responsibilities}

565 Sergio Marconi and Sarah Graves designed the experiment, Sarah Graves carried out field work,

566 Sergio Marcon, Sarah Graves and Ethan White developed the methods, Sergio Marconi

567 performed the analysis, Ethan White and Stephanie Bohlman supervised the work as lab leaders,

568 helped with experimental design and advice on data analysis. Ben Weinstein helped with

569 technical aspects and manuscript editing. All authors contributed to editing the manuscript. We

570 acknowledge Dr. Adytia Singh for his suggestions in the initial phase of the project, and the

571 reviewers that contributed to significantly improve this manuscript. 
574 1. Albert, C.H., Thuiller, W., Yoccoz, N.G., Soudant, A., Boucher, F., Saccone, P. and 575 Lavorel, S., 2010. Intraspecific functional variability: extent, structure and sources of 576 variation. Journal of Ecology, 98(3), pp.604-613. fuel parameters using LIDAR data. Remote sensing of Environment, 94(4), pp.441-449. Muller $\square$ Landau, H.C., Joseph Wright, S., Abu Salim, K., Almeyda Zambrano, A.M., Alonso, A., Baltzer, J.L. and Basset, Y., 2015. CTFS $\square$ ForestGEO: a worldwide network monitoring forests in an era of global change. Global change biology, 21(2), pp.528-549.

1. Asner, G.P., Martin, R.E., Knapp, D.E., Tupayachi, R., Anderson, C.B., Sinca, F., Vaughn, N.R. and Llactayo, W., 2017. Airborne laser-guided imaging spectroscopy to map forest trait diversity and guide conservation. Science, 355(6323), pp.385-389. scale climatic and geophysical controls on the leaf economics spectrum. Proceedings of the National Academy of Sciences, 113(28), pp.E4043-E4051. tropical forests. Ecological Applications, 21(1), 85-98. 
6. Audebert, B. Le Saux and S. Lefevre, "Deep Learning for Classification of Hyperspectral Data: A Comparative Review," in IEEE Geoscience and Remote Sensing Magazine, vol. 7, no. 2, pp. 159-173, June 2019, doi: 10.1109/MGRS.2019.2912563.

7. Bannister, J.M., Herbert, E.R. and Craft, C.B., 2015. Spatial variability in sedimentation, carbon sequestration, and nutrient accumulation in an alluvial floodplain forest. In The Role of Natural and Constructed Wetlands in Nutrient Cycling and Retention on the

8. Barbosa, J.M. and Asner, G.P., 2017. Prioritizing landscapes for restoration based on spatial patterns of ecosystem controls and plant-plant interactions. Journal of Applied Ecology, 54(5), pp.1459-1468.

9. Barnes, R., Lehman, C., Mulla, D., 2014. Priority-flood: An optimal depression-filling and watershed-labeling algorithm for digital elevation models. Computers \& Geosciences 62, 117-127. doi:10.1016/j.cageo.2013.04.024 Computational Statistics \& data analysis, 48(1), pp.17-46. photogrammetry and remote sensing, 65(1), pp.2-16. enhanced decomposition of plant biomass in a freshwater wetland. Aquatic Botany, 127, pp.44-52. 
12. M. Han, Y., Bovolo, F. and Bruzzone, L., 2017. Segmentation-based fine registration of very high resolution multitemporal images. IEEE Transactions on Geoscience and Remote Sensing, 55(5), pp.2884-2897. interactions among plants. Ecology, 84(5), pp.1115-1128.

14. Carmona, C.P., De Bello, F., Mason, N.W. and Lep̌̌, J., 2016. Traits without borders: integrating functional diversity across scales. Trends in ecology \& evolution, 31(5), pp.382-394.

15. Cassey, P. and Blackburn, T.M., 2006. Reproducibility and repeatability in ecology. BioScience, 56(12), pp.958-959. foliar traits in lowland tropical forests. Remote Sensing, 8(2), p.87.

17. Christoffersen, P.F., 1998. Evaluating interval forecasts. International economic review, pp.841-862.

18. Clark, J.S., Gelfand, A.E., Woodall, C.W. and Zhu, K., 2014. More than the sum of the species at ecosystem scales using support vector machine classification and BRDF 
correction on airborne hyperspectral and LiDAR data. Remote Sensing, 4(11), pp.34623480. Ciais, and G. Matteucci., 2019. The sensitivity of the forest carbon budget shifts across processes along with stand development and climate change. Ecological Applications 00(0): e01837. 10.1002/eap.1837 Poorter. 2003. A handbook of protocols for standardised and easy measurement of plant from airborne laser scanning and hyperspectral data. Methods in ecology and evolution, populations, communities and ecosystems. CRC Press.

25. Diaz et al, 2016, The global spectrum of plant form and function. Nature 529:167-171. 10.1038/nature16489. drivers of biodiversity: The importance of trait covariance in community assembly. Ecology letters, 20(7), pp.872-882. 
27. Feilhauer, H., Asner, G.P. and Martin, R.E., 2015. Multi-method ensemble selection of spectral bands related to leaf biochemistry. Remote Sensing of Environment, 164, pp.5765. Squares Regression for Generalized Linear Models, R package version 1.1.1. approach to biomass estimation from remote sensing data in a tropical agricultural landscape. Remote sensing of environment, 218, pp.32-43.

30. Graves, S.J., Asner, G.P., Martin, R.E., Anderson, C.B., Colgan, M.S., Kalantari, L. and Bohlman, S.A., 2016. Tree species abundance predictions in a tropical agricultural landscape with a supervised classification model and imbalanced data. Remote Sensing,

31. Graves S, Gearhart J, Caughlin TT, Bohlman S. 2018. A digital mapping method for linking high-resolution remote sensing images to individual tree crowns. PeerJ Preprints6:e27182v1 https://doi.org/10.7287/peerj.preprints.27182v1 Kaufmann. 
34. Houborg, R., Fisher, J.B. and Skidmore, A.K., 2015. Advances in remote sensing of vegetation function and traits.

35. Jakubowski,K., Q. Guo, and M. Kelly. 2013. Tradeoffs between lidar pulse density and forest measurement accuracy. Remote Sensing of Environment 130:245-253.

36. Jucker, T., Avăcări $\square$ ei, D., Bărnoaiea, I., Duduman, G., Bouriaud, O. and Coomes, D.A., 2016. Climate modulates the effects of tree diversity on forest productivity. Journal of Ecology, 104(2), pp.388-398. comparison of individual tree detection and extraction using airborne laser airborne LiDAR: Comparing sensors and resolutions in a temperate broadleaf forest ecosystem. Forest Ecology and Management, 433, pp.364-375. Werner, G.D., Aakala, T., Abedi, M. and Acosta, A.T., 2020. TRY plant trait databaseHannah, L., Klausmeyer, K., Aldous, A. and Bienz, C., 2010. Resource management in a 
40. Li, X., Messina, J.P., Moore, N.J., Fan, P. and Shortridge, A.M., 2017. MODIS land cover uncertainty in regional climate simulations. Climate Dynamics, 49(11-12),

696 pp.4047-4059.

41. Liu, X., Swenson, N.G., Lin, D., Mi, X., Umaña, M.N., Schmid, B. and Ma, K., 2016. Linking individual $\square$ level functional traits to tree growth in a subtropical forest. Ecology,

42. Ma, X., Mahecha, M.D., Migliavacca, M., van der Plas, F., Benavides, R., Ratcliffe, S., functional diversity from space: the potential of Sentinel-2. Remote Sensing of

43. Marconi, S., Graves, S.J., Gong, D., Nia, M.S., Le Bras, M., Dorr, B.J., Fontana, P., Gearhart, J., Greenberg, C., Harris, D.J. and Kumar, S.A., 2018. A data science challenge Environment, 233, p.111368. generalizable method for remote sensing of canopy nitrogen across a wide range of forest ecosystems. Remote Sensing of Environment 112:3511-3519. Asner, G.P., 2018. An approach for foliar trait retrieval from airborne imaging spectroscopy of tropical Forests. Remote Sensing, 10(2), p.199. 
46. McGill, B.J., Enquist, B.J., Weiher, E. and Westoby, M., 2006. Rebuilding community ecology from functional traits. Trends in ecology \& evolution, 21(4), pp.178-185.

47. Messier, J., McGill, B.J., Enquist, B.J. and Lechowicz, M.J., 2017. Trait variation and integration across scales: is the leaf economic spectrum present at local scales?. Ecography, 40(6), pp.685-697.

48. Milner $\square$ Gulland, E.J. and Shea, K., 2017. Embracing uncertainty in applied ecology. Journal of applied ecology, 54(6), pp.2063-2068. allocation patterns in 27 species of herbaceous plants. Perspectives in plant ecology,

50. Murphy, J., and J. P. Riley. 1962. A modified single solution method for the evolution and systematics, 3(2), pp.115-127.

51. National Ecological Observatory Network. 2014. Data Products NEON.DP1.10098, NEON.DP1.30010, NEON.DP3.30015, NEON.DP1.30008. Provisional data determination of phosphate in natural waters. Analytica Chimica Acta 27:31 - 36 . downloaded from http://data.neonscience.org on 26 Jan 2016. Battelle, Boulder, CO, USA 
Plant phenotypic plasticity in a changing climate. Trends in plant science, 15(12), pp.684692.

54. Niinemets, Ü., Keenan, T.F. and Hallik, L., 2015. A worldwide analysis of within $\square$ canopy variations in leaf structural, chemical and physiological traits across plant functional types. New Phytologist, 205(3), pp.973-993. \& Sons, Ltd (Ed.). doi:10.1002/9780470015902.a0026282

56. Pollock, L.J., Morris, W.K. and Vesk, P.A., 2012. The role of functional traits in species distributions revealed through a hierarchical model. Ecography, 35(8), pp.716-725. and Multispectral Data Fusion with Local Filtering and Variable Window Size for Estimating Tree Height. Photogrammetric Engineering and Remote Sensing. 70. 589-

58. Poorter, H., Niinemets, Ü., Poorter, L., Wright, I.J. and Villar, R., 2009. Causes and 604. 10.14358/PERS.70.5.589. consequences of variation in leaf mass per area (LMA): a meta $\square$ analysis. New 2016. Functional traits shape size-dependent growth and mortality rates of dry forest tree species. Journal of Plant Ecology, 10(6), pp.895-906.

60. Real, R. and Vargas, J.M., 1996. The probabilistic basis of Jaccard's index of similarity. Systematic biology, 45(3), pp.380-385. 
61. Rezatofighi, H., Tsoi, N., Gwak, J., Sadeghian, A., Reid, I. and Savarese, S., 2019. Generalized intersection over union: A metric and a loss for bounding box regression. In Proceedings of the IEEE Conference on Computer Vision and Pattern Recognition (pp. 658-666).

62. Roussel, J.R. and Auty, D., 2017. lidR: Airborne LiDAR Data Manipulation and Visualization for Forestry Applications. issues with PLS and CBSEM: Where the bias lies!. Journal of Business Research, 69(10), pp.3998-4010. Channan, S. and Townshend, J.R., 2015. A model for the propagation of uncertainty from continuous estimates of tree cover to categorical forest cover and change. Remote Sensing of Environment, 156, pp.418-425. Jack, S.B., Gonzalez-Benecke, C., Lee, H., Falkowski, M.J. and Khosravipour, A., 2016. Imputation of individual Longleaf Pine (Pinus palustris Mill.) Tree attributes from field and LiDAR data. Canadian journal of remote sensing, 42(5), pp.554-573. spectroscopy algorithms for mapping canopy foliar chemical and morphological traits and their uncertainties. Ecological Applications, 25(8), pp.2180-2197. 
67. Song, L., Langfelder, P. and Horvath, S., 2013. Random generalized linear model: a highly accurate and interpretable ensemble predictor. BMC bioinformatics, 14(1), p.5.

68. Sumbul, G., Cinbis, R.G. and Aksoy, S., 2019. Multisource region attention network for fine-grained object recognition in remote sensing imagery. IEEE Transactions on Geoscience and Remote Sensing, 57(7), pp.4929-4937.

69. Swenson, N.G. and Weiser, M.D., 2010. Plant geography upon the basis of functional traits: an example from eastern North American trees. Ecology, 91(8), pp.2234-2241. squares. The American Statistician, 54(2), pp.116-118. G. and He, H., 2018. Patterns of plant carbon, nitrogen, and phosphorus concentration in relation to productivity in China's terrestrial ecosystems. Proceedings of the National

72. Valladares, F., Matesanz, S., Guilhaumon, F., Araújo, M.B., Balaguer, L., Benito $\square$ Garzón, M., Cornwell, W., Gianoli, E., Kleunen, M., Naya, D.E. and Nicotra, Academy of Sciences, 115(16), pp.4033-4038. fires." Forest Ecology and Management 258.11 (2009): 2467-2474. 
74. Walters, Michael B., and John P. Gerlach. "Intraspecific growth and functional leaf trait responses to natural soil resource gradients for conifer species with contrasting leaf habit." Tree physiology 33.3 (2013): 297-310.

75. Weinstein, B., Marconi, S., Bohlman, S., Zare, A. and White, E., 2019. Individual treecrown detection in RGB imagery using self-supervised deep learning neural networks. bioRxiv, p.532952. Cavender-Bares, J., Chapin, T., Cornelissen, J.H., Diemer, M. and Flexas, J., 2004. The worldwide leaf economics spectrum. Nature, 428(6985), p.821.

77. Yang, J., Cao, M. and Swenson, N.G., 2018. Why functional traits do not predict tree demographic rates. Trends in ecology \& evolution, 33(5), pp.326-336.

78. Yao, W., Krzystek, P. and Heurich, M., 2012. Tree species classification and estimation of stem volume and DBH based on single tree extraction by exploiting airborne fullwaveform LiDAR data. Remote Sensing of Environment, 123, pp.368-380. crown detection and delineation-evolution of lidar data. Remote Sensing, 8(4), p.333. 
812 Table 1. Data products and sources (National Ecological Observatory Network, 2016).

813 Information about data products can be found on the NEON data products catalogue

814 (http://data.neonscience.org/data-product-catalog).

\begin{tabular}{|c|c|c|c|}
\hline Name & $\begin{array}{l}\text { NEON data } \\
\text { product ID }\end{array}$ & Data date & How it was used \\
\hline $\begin{array}{l}\text { Spectrometer orthorectified at- } \\
\text { sensor radiance }\end{array}$ & $\begin{array}{l}\text { NEON.DP1.3000 } \\
8\end{array}$ & $\begin{array}{l}2014 \text { and } \\
2015\end{array}$ & $\begin{array}{l}\text { Hyperspectral images used to } \\
\text { model foliar chemical and } \\
\text { physical properties }\end{array}$ \\
\hline $\begin{array}{l}\text { Discrete return LiDAR point } \\
\text { cloud }\end{array}$ & $\begin{array}{l}\text { NEON.DP1.300 } \\
03.001\end{array}$ & $\begin{array}{l}2014 \text { and } \\
2015\end{array}$ & $\begin{array}{l}\text { Crown segmentation and } \\
\text { calculation of tree height }\end{array}$ \\
\hline $\begin{array}{l}\text { AOP L2 and L3 data products } \\
\text { (Albedo, Elevation, Slope, } \\
\text { Aspect) }\end{array}$ & $\begin{array}{l}\text { NEON.DP2.3001 } \\
2.001, \\
\text { DP3.30024.001, } \\
\text { DP3.30025.001 }\end{array}$ & $\begin{array}{l}2014 \text { and } \\
2015\end{array}$ & $\begin{array}{l}\text { Link modeled tree crown } \\
\text { properties with other NEON- } \\
\text { AOP data products }\end{array}$ \\
\hline $\begin{array}{l}\text { Woody plant vegetation } \\
\text { structure }\end{array}$ & DP1.10098.001 & $\begin{array}{l}2014- \\
2018\end{array}$ & $\begin{array}{l}\text { Build allometric relationship to } \\
\text { infer DBH from Crown Area and } \\
\text { Tree Height }\end{array}$ \\
\hline Field ITC & $\begin{array}{l}\text { Graves et al. } \\
2018\end{array}$ & 2017 & $\begin{array}{l}\text { Validate crown segmentation, } \\
\text { define tree objects. }\end{array}$ \\
\hline
\end{tabular}

815 


\section{Figures captions}

817 Figure 1. Workflow of the pipeline following Unified Modeling Language (UML). Left side

818 shows the method to build and test three modeling approaches: PBM (pixel-based model), EPBS

819 (ensemble of pixel-based models), CEAM (crown ensemble averaged model). Right side

820 represents the part of the pipeline dealing with scaling, fusion, and public distribution of derived

821 data. NEON L3 products fused to the derived traits data are: Aspect (DP3.30025.001), Elevation

822 (DP3.30024.001), and Slope (DP3.30025.001).

823

824

825

826

827

828

829

830

831

832

833

834 extracted from algorithmically delineated crowns; $(C)$ difference in performance (RMSE)

835 between models tested on pixels extracted from field and algorithmically delineated crowns.

836 Positive values mean that for that comparison model built on automatically delineated crowns

837 performed better. (D) Coverage of the 95 predictions intervals on held out data for the four 
838 models (LMA, N, P and C). Dash-dotted line represent the ideal coverage value of 95\%.

839 Figure 4. Example of predictions at the landscape scale for individual tree crowns (ITC) at

840 Ordway Swisher Biological Station (OSBS). In the center, predictions of tree height for $\sim 2.5$

841 million trees within NEON AOP footprint (215 km2), plotted in a quantile scale using the viridis

842 color palette. Cropped images represent a 1 $\mathrm{km} 2$ detail of LMA, \%N, \%C, and \%P predictions

843 at scale. Expected values are presented on a quantile scale using a spectral color palette (with

844 lower values in red, and higher values in blue). Range of the 95\% probability intervals for the

845 same area are presented on the intensity scale of blues (with lower values in white, and higher

846 values in deep blue).

848 Figure 5. Leaf chemical distributions and relationship with tree height on a random sample of

849 100,000 derived individual tree crowns (ITCs). On the top row, comparison between

850 distributions of C, LMA, \%P, and \%N for the two sites, OSBS (blue), and TALL (yellow).

851 Vertical dotted lines represent the average for the site. Rug plots on the $x$-axis represent the

852 marginal distribution of OSBS and TALL data between the minimum and maximum range of

853 derived observations. On the lower row, example of relationship between tree height and the

854 same three leaf chemical traits: from left to right, LMA, \%N, \%P. Linear trends and 95CI

855 ellipses are represented for each relationship and site, following the same color scheme as

856 above. 


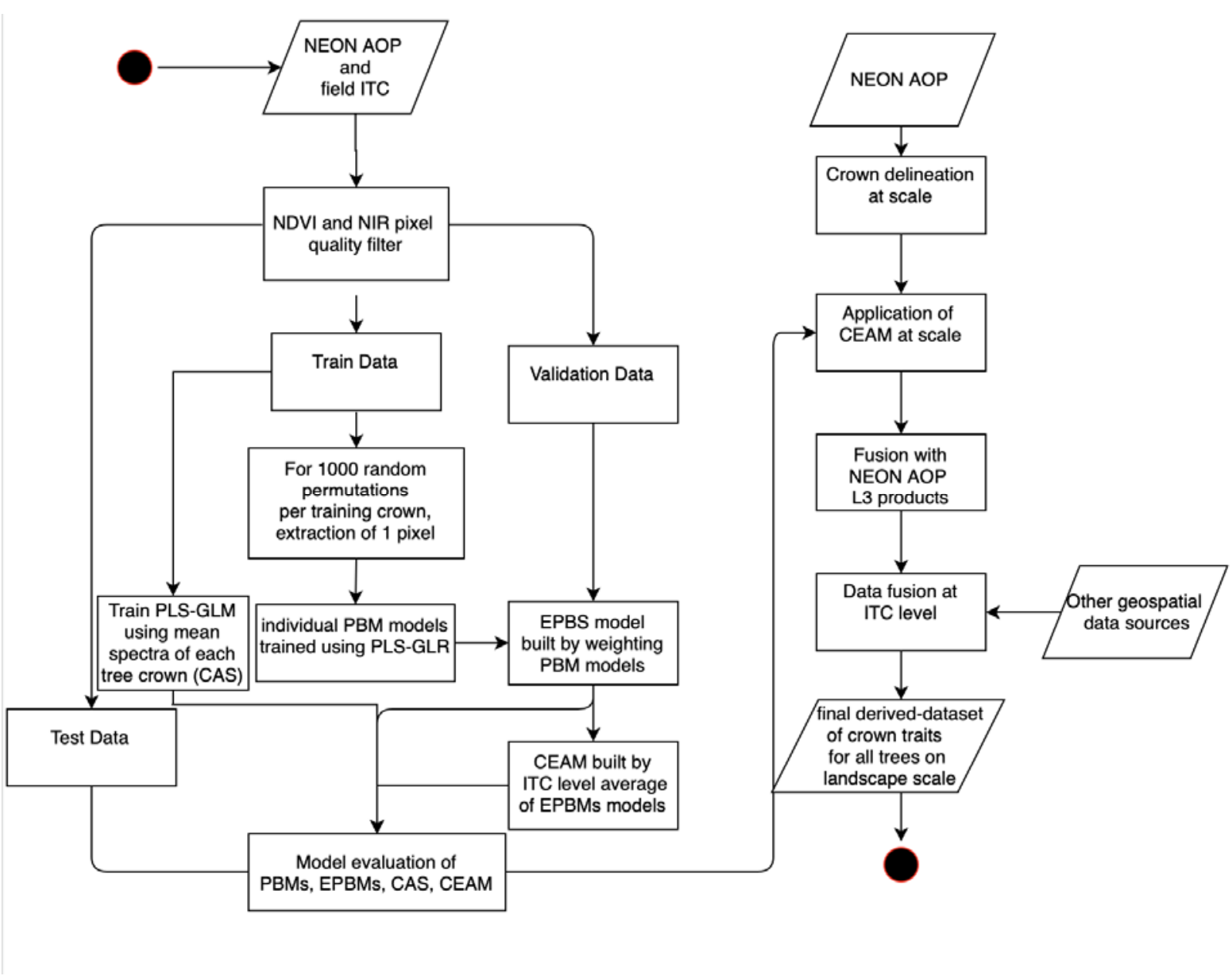

861 Figure 1. Workflow of the pipeline following Unified Modeling Language (UML). Left side

862 shows the method to build and test three modeling approaches: PBM (pixel-based model), EPBS

863 (ensemble of pixel-based models), CEAM (crown ensemble averaged model). Right side

864 represents the part of the pipeline dealing with scaling, fusion, and public distribution of derived

865 data. NEON L3 products fused to the derived traits data are: Aspect (DP3.30025.001), Elevation

866 (DP3.30024.001), and Slope (DP3.30025.001). 

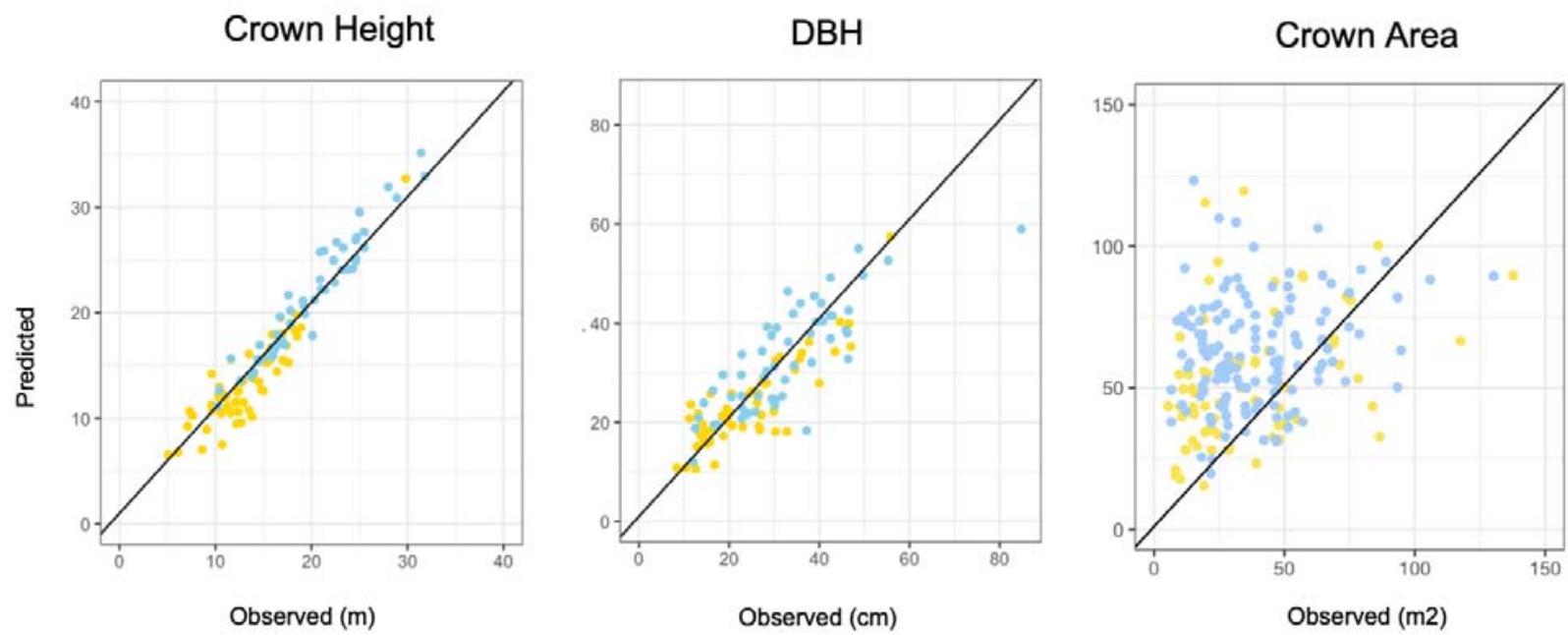

870 Figure 2. Comparison between observed and predicted tree structural traits for algorithmically

871 delineated crowns corresponding to ground delineated ITCs: $(A)$ tree crown height (m), (B) tree

872 diameter at breast height $(\mathrm{cm})$, and $(C)$ tree crown area $(\mathrm{m} 2)$. Yellow and blue points represent

873 OSBS and TALL site data points respectively. Black diagonal is the 1:1 line.

874

875

876 

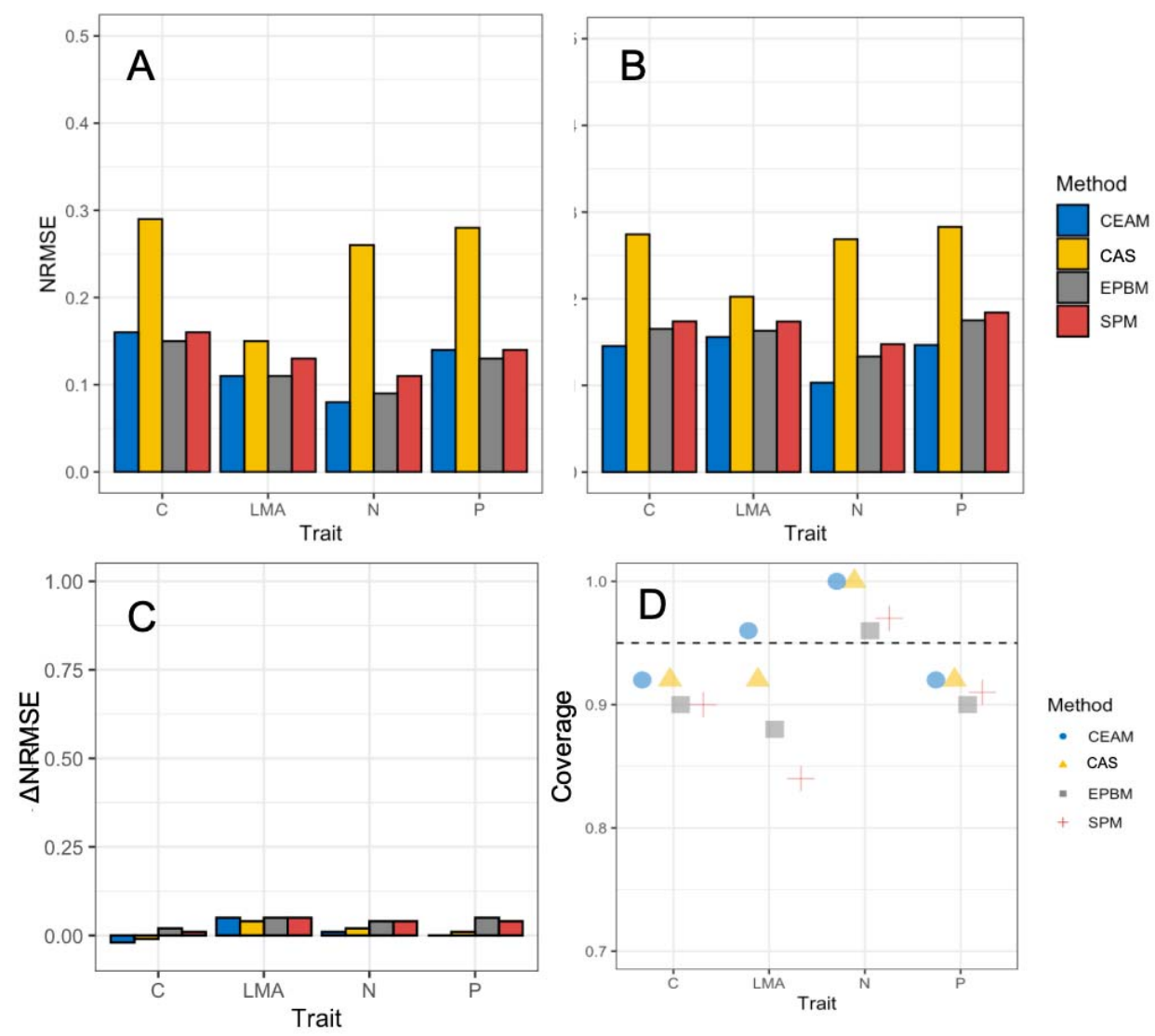

Figure 3. Model evaluation and comparison of pixel based (SPM, red), ensemble pixels (EPBM,

879 grey), crown spectra average (CAS, yellow), and crown ensemble average (CEAM, blue)

880 predictions on an independent test set of 24 observed crowns for \%P, LMA, \%N and \%C. (A)

881 Evaluation and comparison of RMSE from models built on pixels extracted from ground

882 delineated crowns; (B) Evaluation and comparison of RMSE from models built on pixels

883 extracted from algorithmically delineated crowns; $(C)$ difference in performance (RMSE)

884 between models tested on pixels extracted from field and algorithmically delineated crowns.

885 Positive values mean that for that comparison model built on automatically delineated crowns

886 performed better. (D) Coverage of the 95 predictions intervals on held out data for the four

887 models (LMA, N, P and C). Dash-dotted line represent the ideal coverage value of 95\%. 


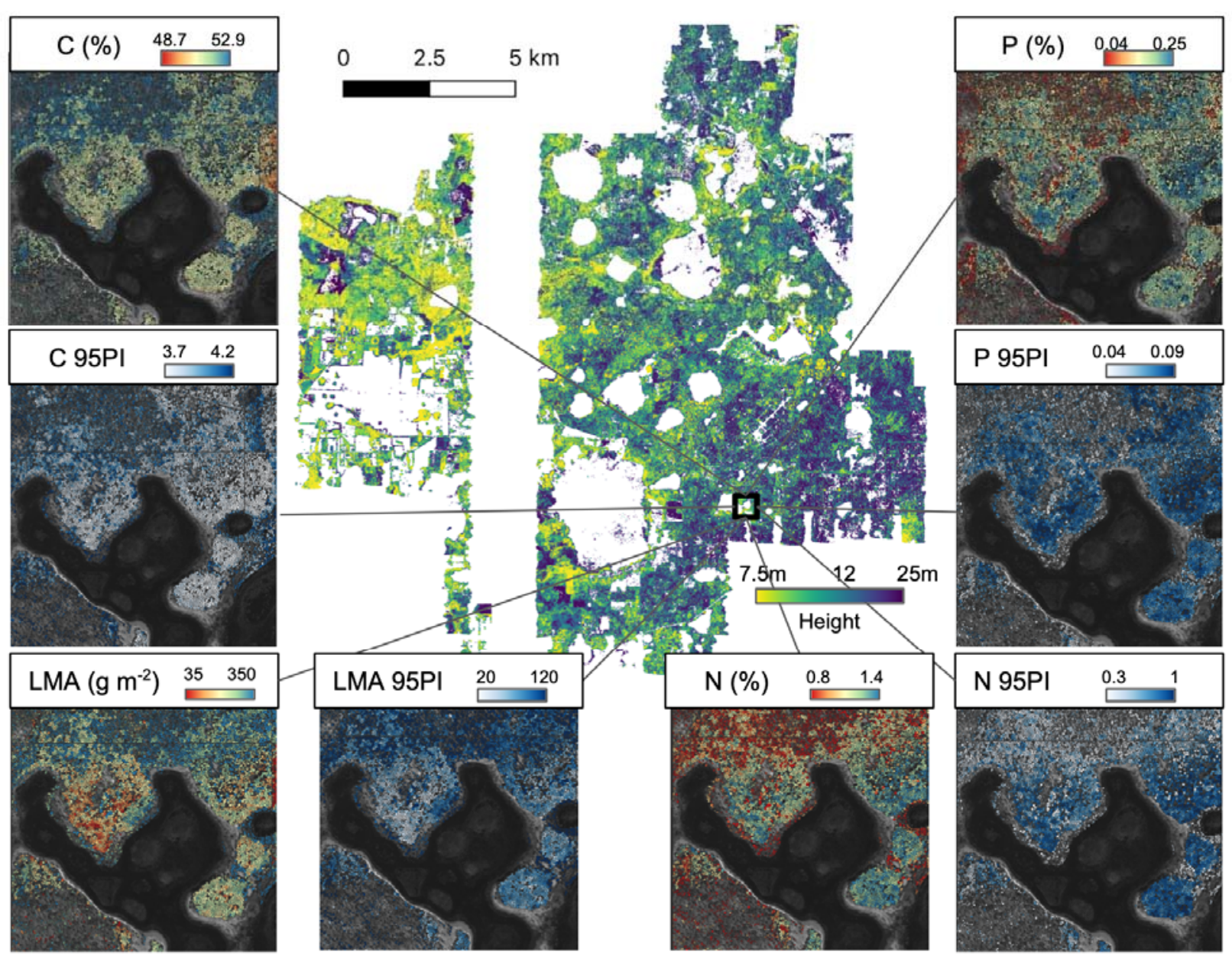

891 Figure 4. Example of predictions at the landscape scale for individual tree crowns (ITC) at

892 Ordway Swisher Biological Station (OSBS). In the center, predictions of tree height for $\sim 2.5$

893 million trees within NEON AOP footprint (215 km2), plotted in a quantile scale using the viridis

894 color palette. Cropped images represent a $\sim \mathrm{km} 2$ detail of $L M A, \% \mathrm{~N}, \% \mathrm{C}$, and \%P predictions

895 at scale. Expected values are presented on a quantile scale using a spectral color palette (with

896 lower values in red, and higher values in blue). Range of the 95\% probability intervals for the

897 same area are presented on the intensity scale of blues (with lower values in white, and higher

898 values in deep blue). 

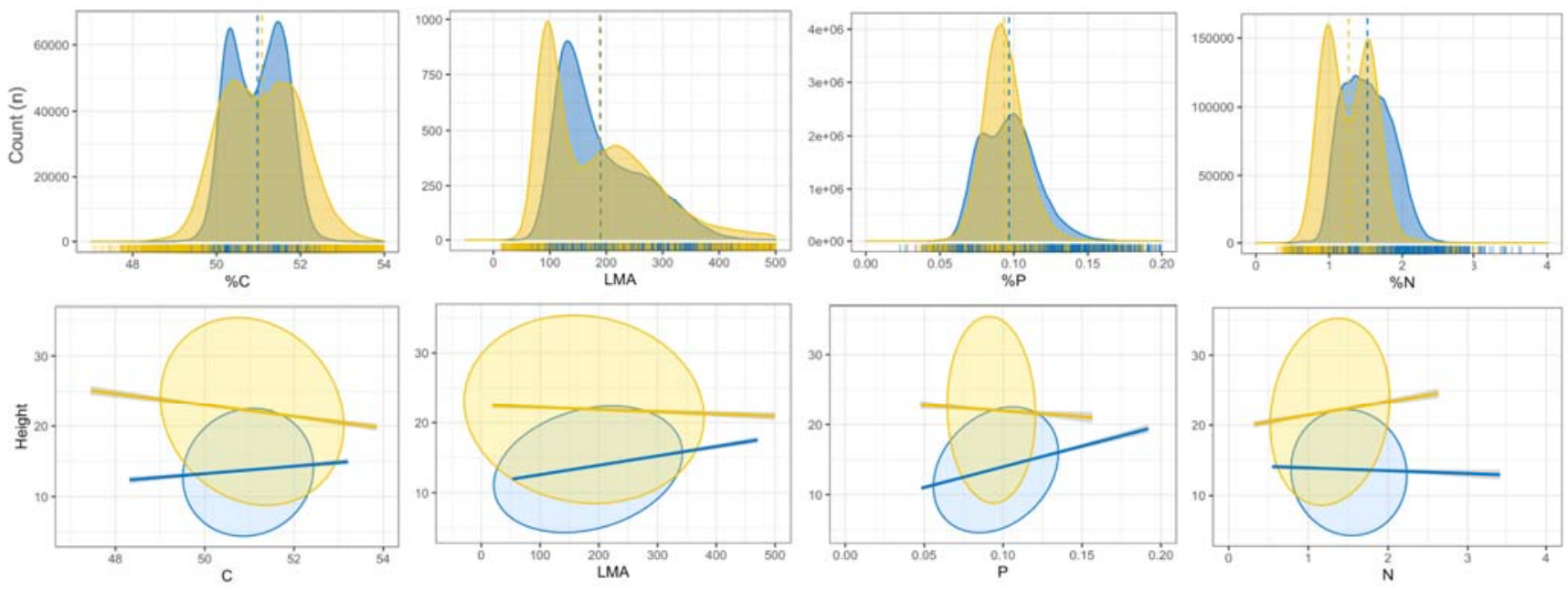

901 Figure 5. Leaf chemical distributions and relationship with tree height on a random sample of

902 100,000 derived individual tree crowns (ITCs). On the top row, comparison between

903 distributions of C, LMA, \%P, and \%N for the two sites, OSBS (blue), and TALL (yellow).

904 Vertical dotted lines represent the average for the site. Rug plots on the $x$-axis represent the

905 marginal distribution of OSBS and TALL data between the minimum and maximum range of

906 derived observations. On the lower row, example of relationship between tree height and the

907 same three leaf chemical traits: from left to right, LMA, \%N, \%P. Linear trends and 95CI

908 ellipses are represented for each relationship and site, following the same color scheme as

909 above.

910

911 